\title{
The Electric Field Calculation for Mobile Communication Coverage in Buildings and Indoor Areas by Using the Method of Auxiliary Sources
}

\author{
Vasil Tabatadze $\mathbb{D}^{10},{ }^{1,2}$ Kamil Karaçuha, ${ }^{1}$ Eldar Veliyev, ${ }^{1,3}$ Ertuğrul Karaçuha, \\ and Revaz Zaridze ${ }^{2}$ \\ ${ }^{1}$ Informatics Institute of Istanbul Technical University, Maslak-34469, Istanbul, Turkey \\ ${ }^{2}$ Tbilisi State University, 1 Chavchavadze Avenue, Tbilisi 0179, Georgia \\ ${ }^{3}$ NTU Kharkiv Polytechnic Institute, Kharkiv 61000, Ukraine
}

Correspondence should be addressed to Vasil Tabatadze; vasilitabatadze@gmail.com

Received 3 October 2019; Accepted 10 March 2020; Published 8 April 2020

Academic Editor: Qingling Wang

Copyright (C) 2020 Vasil Tabatadze et al. This is an open access article distributed under the Creative Commons Attribution License, which permits unrestricted use, distribution, and reproduction in any medium, provided the original work is properly cited.

In this article, the diffraction of the electromagnetic wave by the building with two rooms is considered. The rooms have doors and windows with lossy dielectric walls. The electromagnetic properties of the building as an opened coupled resonator system are investigated at different source locations and several frequencies including $5 \mathrm{G}$ band. The problem is solved by using the Method of Auxiliary Sources. The near electric field distributions are calculated and analyzed.

\section{Introduction}

Nowadays, one of the forecasts in the studies carried out on the predictions for the future is that the connectivity will increase [1]. This has been already seen in our daily lives. Access to the information and demand to disseminate information is increasing rapidly. The development of a new generation electronic communication systems to meet this demand continues rapidly. In this context, especially mobile communication systems come to the agenda with a new generation every 10 years. For example, 2G, 3G, 4G, and 5G. These developments lead to the frequency band demand which can be thought of as a scarce and limited resource in the telecommunication sector. For 5G, the installed systems require the use of higher frequency bands. As it is known, the attenuation of electromagnetic waves at higher frequencies is greater than the lower frequencies in free space. For example, take an example of $900 \mathrm{MHz}$ and $1800 \mathrm{MHz}$ frequencies, it is necessary to install more base stations in the $1800 \mathrm{MHz}$ band to cover the same geographical area.

Very soon, 5G mobile communications systems will be commercially available. It is commonly known herein that the band at $3.6 \mathrm{GHz}$ is intended to be introduced in Europe [2]. This band will increase the use of more base stations both in open and closed areas (motorways, shopping centers, workplaces, hospitals, etc.). Therefore, this will significantly increase operator costs. In this study, we aim to investigate the electromagnetic wave propagation at 300, 450, 900, 1800, and $3600 \mathrm{MHz}$ in a closed area and try to find the field at any point. This will contribute to the establishment of a costeffective system for the installation location of the base stations that will provide the appropriate coverage.

The coverage problem of the mobile phone or base station radiation is important and an open question not only for companies but also for researchers because the complicated geometry of the building (two rooms) and the presence of windows and doors do not give strong and persuasive intuition to predict the field distribution. Therefore, it is not an easy task, initially, to determine where the radiation source should be put to have better coverage in both rooms. In other words, the signal strength should not be very different. Several works are carried out in this direction. Some works use the ray tracing to evaluate reflected rays [3-7]. 
Our work has a novel approach based on the Method of Auxiliary Sources (MAS) which gives the ability to realize full-wave simulation. Full-wave simulation is a better approximation to the real scenario. In previous works $[8,9]$, the case of the human body located in the metallic room with transparency was investigated. On the contrary, the study carried out in this paper takes into account the wall as the lossy dielectric medium.

First, theoretical part based on solving the diffraction problem of the electromagnetic waves by the building with two rooms is given; then, based on this mathematical algorithm, the program package is created which gives the ability to change the following parameters such as the building geometry, in particular, the wall thickness, dimensions of the rooms, the location and dimensions of the doors and windows, and the electrical parameters of the walls such as permittivity and conductivity. Furthermore, the source of the radiation can be inside or outside the building. Both cases for mobile phone radiation and the base station radiation can be considered. Our goal here is to show that our approach visualizes the field distribution inside the building and the program package can be used by the people who decide where to put the radiation source in the building to have acceptable and satisfactory coverage. We conducted several numerical experiments and the obtained results are presented in this paper with the corresponding analysis.

In our problem, there can be asymmetric room configurations. For ray tracing, the solution would be much complicated. However, in the proposed method, there is not much difference between asymmetry and symmetry in the sense of complexity for the solution of the problem.

\section{Formulation of the Problem}

In this study, the electric field distribution for a building with double rooms is investigated. In order to find the field distribution in the rooms, a very efficient method called Method of Auxiliary Source is preferred. Approximately for 50 years, MAS is used in the diffraction problems by different researchers including 2 - and 3 -dimensional problems [8-11]. The method also gives the ability to find the near field distributions. In MAS, the unknown scattered field due to the obstacles in the space can be represented as the superposition of the fields created by auxiliary sources with unknown amplitudes for each physical region. The unknown coefficient can be found by applying the boundary condition on the surface of the obstacles or objects. The main advantage of MAS is to locate the specifically arranged auxiliary sources on two imaginary surfaces which are above and below the real surface of the object. In this way, the singularity problem while applying boundary conditions is avoided [8-11]. The imaginary surface locating interior of the obstacle stands for the scattered field outside the obstacle by applying the boundary condition on the real surface, whereas the imaginary surface locating exterior of the obstacle stands for the scattered field inside the obstacle by applying the boundary condition on the real surface. As a summary, the function of the MAS in this problem would be the construction of two couples of closed auxiliary surfaces outside and inside of the dielectric walls corresponding to the scattered field inside and outside of the walls, respectively.

The investigation of the distribution of the electric field in the building with two rooms is carried out by using the Method of Auxiliary Sources (MAS). In Figure 1, the 3dimensional geometry is given. Also, in Figure 2, the section of the top view where the auxiliary sources are added can be seen. Note that, in the geometry of the rooms, the corners are replaced by smooth surfaces because MAS requires smooth surfaces $[10,11]$. Note that the walls are assumed to be lossy dielectric. The program package is created so that the dimension of rooms and the location of the windows and doors can be changed easily. Two auxiliary surfaces that are inside and outside of the main surface are constructed. The inner surface describes the field outside the walls, whereas the outer one describes the field inside the wall. The field outside the wall means both inside the room and outside the room. Each room has one window and one door. The dimensions of the rooms are given in Table 1.

The corner curvature radius of outer and inner surfaces of the room is 0.1 meters, and windows and doors corner curvature is 0.0625 meters. The number of collocation points depends on the frequency. The investigation shows that, in order to get the correct solution, for each $\lambda^{2}$, there should be at least 9 points, where $\lambda$ is the wavelength in the dielectric [8-10]. Dielectric permittivity and conductivity are $\varepsilon=4$, $\sigma=0.05 \mathrm{~S} / \mathrm{m}[12]$.

The dimensions of the building are a bit smaller compared to real-world cases in order to decrease the required computational resources, but the main results and field behavior obtained below will be valid.

On the auxiliary surface, it is distributed as two mutual perpendicular Hertz dipoles. The electric field and the magnetic field are given in (1) and (2), respectively [13]:

$$
\begin{aligned}
\vec{G}_{E}(k \vec{r})= & \frac{1}{4 \pi \varepsilon} e^{i k r}\left\{\frac{1}{R^{3}}\left[3 \vec{r}_{u}\left(\vec{r}_{u} \cdot \vec{P}\right)-\vec{P}\right]\right. \\
& \left.\cdot-\frac{i k}{r^{2}}\left[3 \vec{r}_{u}\left(\vec{r}_{u} \cdot \vec{P}\right)-\vec{P}\right]-\frac{k^{2}}{R}\left[\vec{r}_{u} x\left(\vec{r}_{u} x \vec{P}\right)\right]\right\},
\end{aligned}
$$

$$
\vec{G}_{H}(k \vec{r})=-\frac{i \omega}{4 \pi} e^{i k r}\left(\frac{1}{r^{2}}-\frac{i k}{r}\right) \vec{P} x \vec{r}_{u}
$$

Here, $\vec{r}_{u}$ is a unit vector directed from the dipole towards the observer, and $r$ is the distance between the source point and observation point. The time dependency is the timeharmonic and $e^{-i \omega t}, k$ is the wavenumber in the medium, and $\vec{P}$ is the polarization vector.

$\vec{G}_{E}(k \vec{r})$ and $\vec{G}_{H}(k \vec{r})$ are the electric and magnetic fields of a Hertz dipole, respectively. Both serve as the Green function. The electric and magnetic fields inside and outside of the wall can be calculated by (3) and (4), respectively $[10,11]$ : 


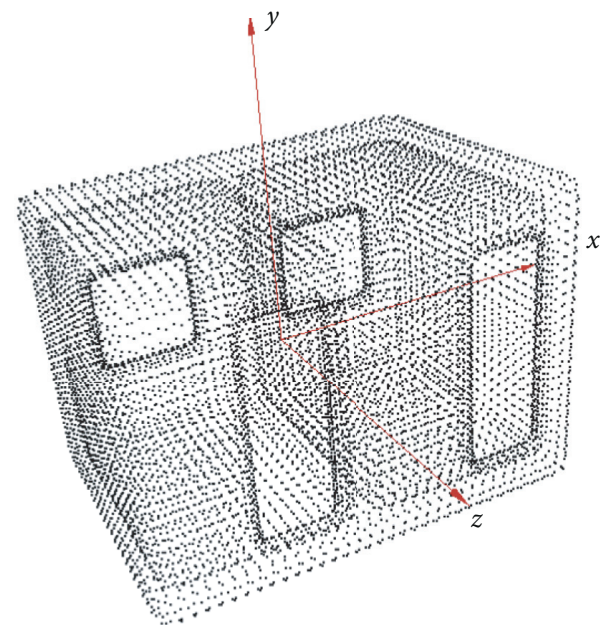

FIGURE 1: The geometry of the building.

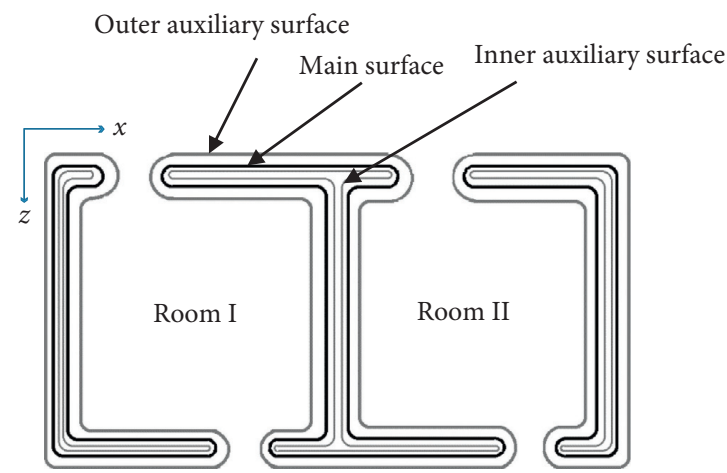

Figure 2: The cross-section ( $x-z$ plane) of the building geometry with inner and outer auxiliary surfaces.

TABLE 1: The dimensions of the rooms.

\begin{tabular}{lcccc}
\hline Building & Height $(\mathrm{m})$ & Width $(\mathrm{m})$ & Length $(\mathrm{m})$ & Thickness of the wall (m) \\
\hline Total & 2.00 & 2.00 & 2.80 & 0.25 \\
Room I & 1.75 & 1.75 & 1.25 & 0.30 (between the rooms) \\
Room II & 1.75 & 1.75 & 1.25 & 0.30 (between the rooms) \\
Door & 1.50 & 1.25 & 0.60 & - \\
Window & 0.80 & 1.25 & 0.80 & - \\
\hline \multicolumn{5}{c}{} \\
$\vec{E}_{\text {in }}(\vec{r})=\sum_{i=1}^{N} X_{1 i}^{\text {out }} G_{E}\left(k_{\text {in }}\left|\vec{r}-\vec{r}_{\text {out }}\right|\right)+\sum_{i=1}^{N} X_{2 i}^{\text {out }} G_{E}\left(k_{\text {in }}\left|\vec{r}-\vec{r}_{\text {out }}\right|\right)$, \\
$\vec{E}_{\text {out }}(\vec{r})=\sum_{i=1}^{N} X_{1 i}^{\text {in }} G_{E}\left(k_{\text {out }}\left|\vec{r}-\vec{r}_{\text {in }}\right|\right)+\sum_{i=1}^{N} X_{2 i}^{\text {in }} G_{E}\left(k_{\text {out }}\left|\vec{r}-\vec{r}_{\text {in }}\right|\right)+\vec{E}_{\text {inc }}(\vec{r})$, \\
$\vec{H}_{\text {in }}(\vec{r})=\sum_{i=1}^{N} X_{1 i}^{\text {out }} G_{H}\left(k_{\text {in }}\left|\vec{r}-\vec{r}_{\text {out }}\right|\right)+\sum_{i=1}^{N} X_{2 i}^{\text {out }} G_{H}\left(k_{\text {in }}\left|\vec{r}-\vec{r}_{\text {out }}\right|\right)$, \\
$\vec{H}_{\text {out }}(\vec{r})=\sum_{i=1}^{N} X_{1 i}^{\text {in }} G_{H}\left(k_{\text {out }}\left|\vec{r}-\vec{r}_{\text {in }}\right|\right)+\sum_{i=1}^{N} X_{2 i}^{\text {in }} G_{H}\left(k_{\text {out }}\left|\vec{r}-\vec{r}_{\text {in }}\right|\right)+\vec{H}_{\text {inc }}(\vec{r})$.
\end{tabular}


Here, $k_{\text {in }}$ is the wavenumber inside the wall and $k_{\text {out }}$ is the wavenumber outside the wall. The locations of the auxiliary sources inside and outside of the wall are given as $\vec{r}_{\text {in }}$ and $\vec{r}_{\text {out }}$, respectively. $\vec{E}_{\text {inc }}(\vec{r})$ and $\vec{H}_{\text {inc }}(\vec{r})$ are the incident electric and magnetic fields, respectively. $\vec{E}_{\text {in }}, \vec{E}_{\text {out }}$, $\vec{H}_{\text {in }}$, and $\vec{H}_{\text {out }}$ are the electric and magnetic fields inside and outside of the wall, respectively. In our case, the excitation is conducted by Hertz dipole with $z$-polarization. The coefficients $X_{1 i}^{\text {out }}$ and $X_{2 i}^{\text {out }}$ are the unknowns of the auxiliary source's amplitude. They should be defined by the boundary conditions:

$$
\begin{aligned}
& \vec{E}_{\tau_{1}}^{\text {in }}\left(\vec{r}_{0}\right)=\vec{E}_{\tau_{1}}^{\text {out }}\left(\vec{r}_{0}\right)+\vec{E}_{\tau_{1}}^{\text {inc }}\left(\vec{r}_{0}\right), \\
& \vec{E}_{\tau_{2}}^{\text {in }}\left(\vec{r}_{0}\right)=\vec{E}_{\tau_{2}}^{\text {out }}\left(\vec{r}_{0}\right)+\vec{E}_{\tau_{2}}^{\text {inc }}\left(\vec{r}_{0}\right), \\
& \vec{H}_{\tau_{1}}^{\text {in }}\left(\vec{r}_{0}\right)=\vec{H}_{\tau_{1}}^{\text {out }}\left(\vec{r}_{0}\right)+\vec{H}_{\tau_{1}}^{\text {inc }}\left(\vec{r}_{0}\right), \\
& \vec{H}_{\tau_{2}}^{\text {in }}\left(\vec{r}_{0}\right)=\vec{H}_{\tau_{2}}^{\text {out }}\left(\vec{r}_{0}\right)+\vec{H}_{\tau_{2}}^{\text {inc }}\left(\vec{r}_{0}\right) .
\end{aligned}
$$

Here, $\vec{r}_{0}$ is the point on the wall that the boundary conditions need to be satisfied. The points are called as the collocation points. $\tau_{1}-$ is the first tangential vector to the building surface and $\tau_{2}-$ is the second tangential vector to the building surface. If the number of collection points is $N$, then, the number of unknowns become $4 \mathrm{~N}$. In order to find all of them, $4 N$ equations are needed. For each point, 4 boundary conditions are required 2 for electric and 2 for magnetic fields, which in total give $4 N$ equations. By solving the system of linear algebraic equations having dimensions of $4 N$ by $4 N$, all unknown coefficients can be found and this gives the ability to find the field values inside or outside of the room and inside the walls.

\section{Numerical Results}

Based on the formulation of the problem section, the program package is created which gives the ability to change the parameters such as the location of the doors and windows, dimension of the room, medium parameters, frequency, source location, and polarization. Before directly presenting the results, the physical properties of the room would be presented. Rooms are partly open dielectric mediums; therefore, for certain frequencies, they act as 3-dimensional open resonators. Because the number of rooms is two, in this study, we deal with two resonators which are coupled, electromagnetically. Usually, the behavior of coupled resonators is much more complicated than separated resonators. The goal of the study is to investigate the coverage of the field inside the room. However, intuitively it is not an easy task to predict the behavior of the field distributions because few changes in the frequency and the source position can redistribute the energy between these rooms. The advantage of the method is to investigate these phenomena in detail. On the contrary, the other methods such as ray tracing method cannot give such a prediction because the resonance is purely a wave phenomenon.

In the following figures, we will take into account different radiation scenarios. For all figures, the total electric field is given and the unit is Volt/meter and the source is transmitting electromagnetic wave having 1 watt power for each case. In particular, the field distribution in both rooms for different frequencies and the source locations are investigated. First, the source is located on the $x$-axis, $100 \mathrm{~m}$ away from the origin and traveling through the opposite direction of the $x$-axis ( $-x$ direction). In Figures $3-7$, the $z$ components of the electric field distribution are given for different frequencies. As a general comment, for each frequency, the field on the $x-y$ plane is highly symmetric but the field on the $x-z$ plane is asymmetric due to having doors and windows. Second, the investigation is carried out on the source location for $3600 \mathrm{MHz}$ frequency given in Figures 8-12.

In the study, the main investigation is to answer how the field is distributed inside and outside the room. In order to understand the coverage of the field, it is assumed that the coverage is good and has an acceptable level when the observation is obtained for the far-zone ( $30 \lambda$ away from the center of the room). Then, the comparison is carried out between the far-field value of the electric field and the average value inside each room. The reason why such an approach is chosen is that the source location can be changed. Therefore, the main goal is to find the effect of the rooms on the distribution of the electric field.

In Figure 3, the field distributions are given for $300 \mathrm{MHz}$. The field in Room II is higher and the field penetrates Room I, barely. For that frequency, openings such as doors and windows do not affect the distribution of the field due to having a large wavelength compared to the dimensions of the doors and windows. In the figure, the amplitudes of the electric field distribution in Room I and Room II, approximately $25 \%$ and $50 \%$ of the amplitude of electric field obtained for the far-zone, respectively.

In Figure 4, the field distributions are given for $450 \mathrm{MHz}$. The field distribution in Room II is higher but also in Room I the field penetrates. In the figure, the amplitudes of the electric field distribution in Room I and Room II, approximately $60 \%$ and $80 \%$ of the amplitude of electric field, are obtained for the far-zone, respectively.

In Figure 5, the field distributions are given for $900 \mathrm{MHz}$. Here, again, the field distribution in Room II is higher. Note that, windows and doors affect the distribution, noticeably. In the figure, the amplitudes of the electric field distribution in Room I and Room II, approximately $70 \%$ and $90 \%$ of the amplitude of electric field, are obtained for the far-zone, respectively.

In Figure 6, the field distributions are given for $1800 \mathrm{MHz}$. The field has a higher value in Room I. This result is very important because it is the reason why the geometry can be assumed to be an open and coupled resonator. The energy is captured by Room I as an open coupled resonator. That is the reason why the amplitude of the electric field inside Room I has higher values compared to far-zone and also Room II. The maximum amplitude of the total electric field in the rooms is approximately $0.3[\mathrm{~V} / \mathrm{m}]$.

In Figure 7, the field distributions are given for $3600 \mathrm{MHz}$ which is proposed 5G frequency for European Zone [2]. The field has a higher value in Room I. In order to 


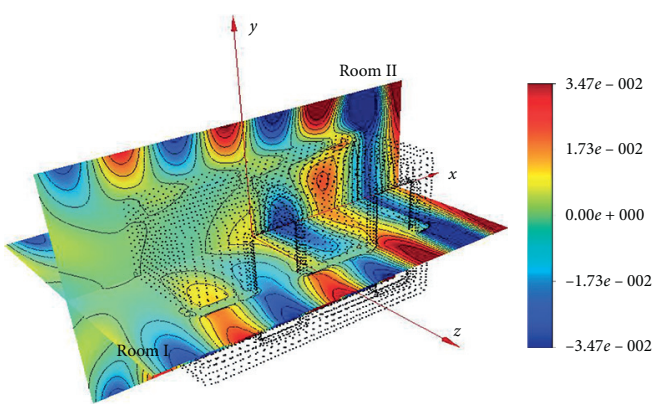

(a)

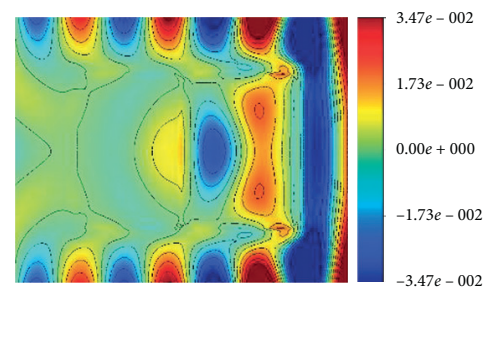

(b)

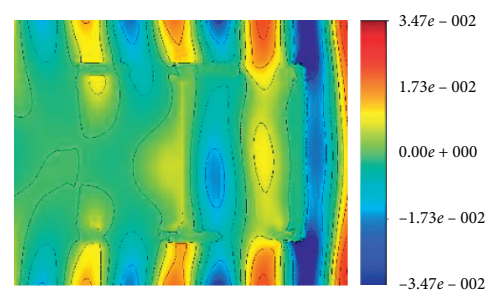

(c)

Figure 3: The isometric (a), $x-y$ plane (b), and $x-z$ (c) plane view of the rooms for $300 \mathrm{MHz}$.

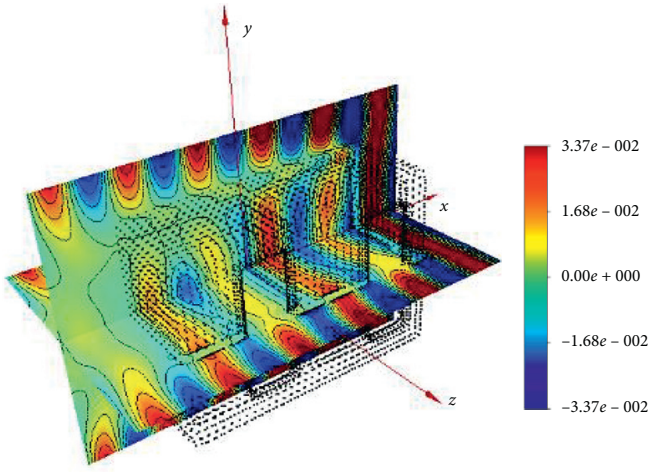

(a)
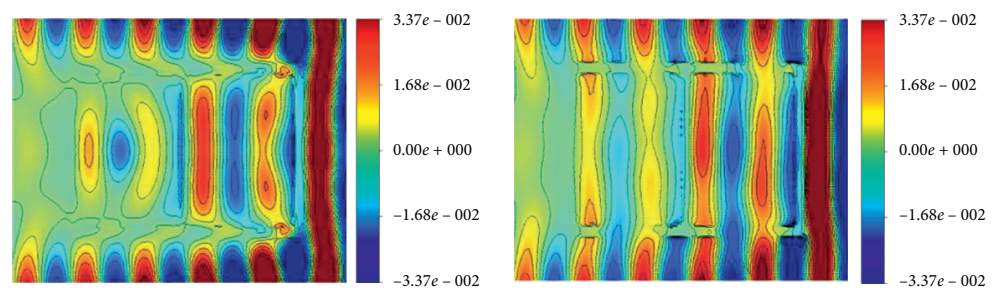

(b) (c)

Figure 4: The isometric (a), $x-y$ plane (b), and $x-z$ plane (c) view of the rooms for $450 \mathrm{MHz}$.

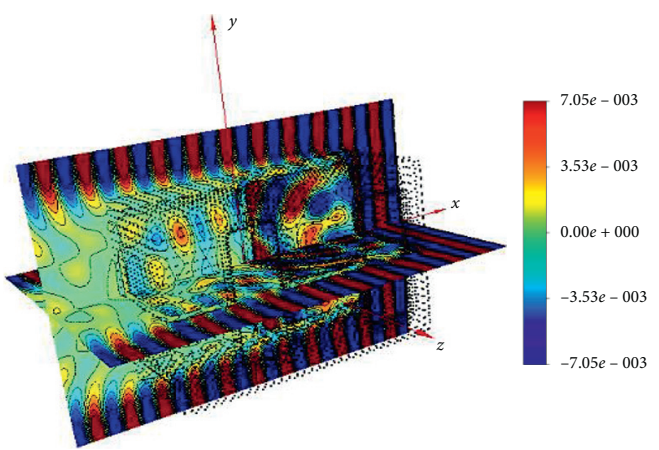

(a)

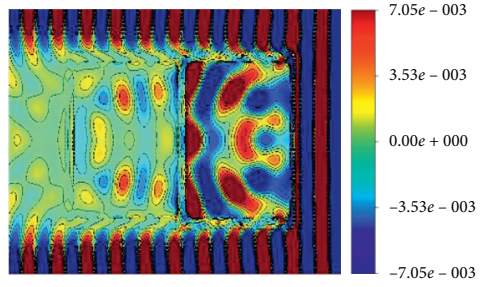

(b)

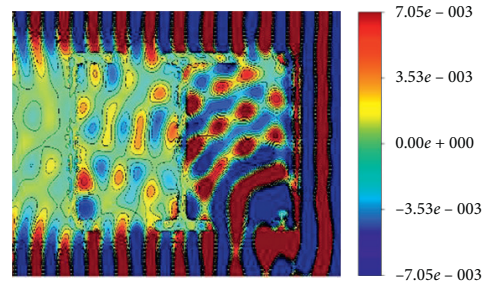

(c)

Figure 5: The isometric (a), $x-y$ plane (b), and $x$ - $z$ plane (c) view of the rooms for $900 \mathrm{MHz}$.

obtain Figure 7, a supercomputer is used. The field distribution is quite uniform on the $x-z$ plane, whereas Room II has higher field amplitude values on the $x-z$ plane. The maximum amplitude of the total electric field in the rooms is approximately $4[\mathrm{~V} / \mathrm{m}]$.

In Figures 8-12, the change of electric field distribution depending on the source location for $3600 \mathrm{MHz}$ is investigated. By this research, the coverage of the $5 \mathrm{G}$ for indoor applications and the optimum location for the source can be studied. As expected, when the source is located inside the room, the amplitude of the electric field increases.

In Figure 8, the source is located at $(1.2 \mathrm{~m}, 0,0)$ which is in Room II and shown as a red circle. In other words, the source is located near the wall of Room II and at the middle in the sense of height and width $(y=0$ and $z=0$ are the planes cutting the rooms equally for the height and the width, respectively). For the figure, the electric field distribution is not uniform in both rooms for $x-y$ plane. Note that 


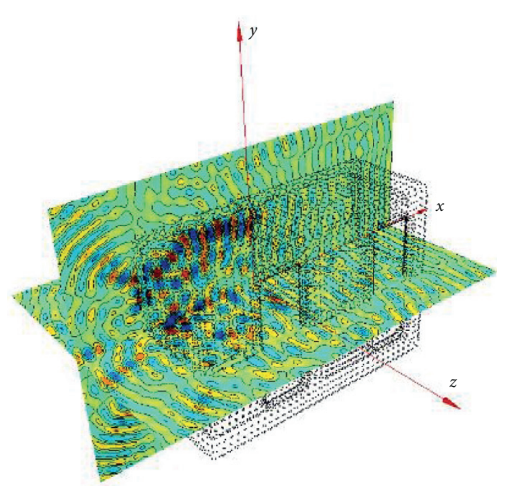

(a)
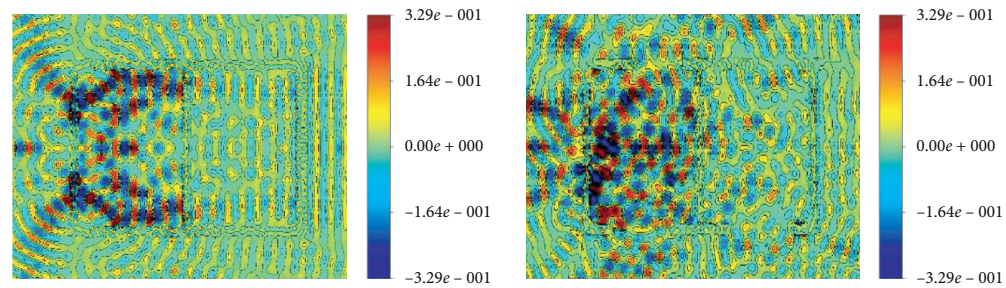

(b) (c)

Figure 6: The isometric (a), $x-y$ plane (b), and $x-z$ plane (c) view of the rooms for $1800 \mathrm{MHz}$.

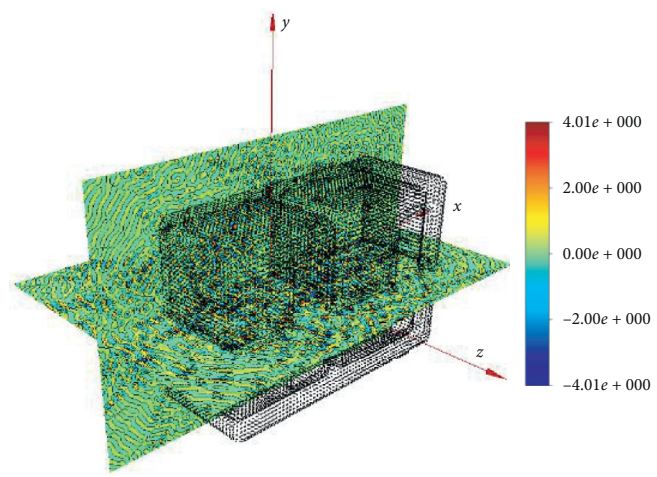

(a)

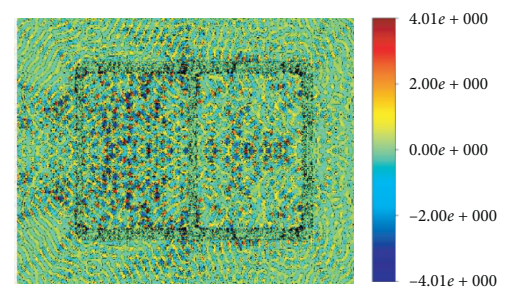

(b)

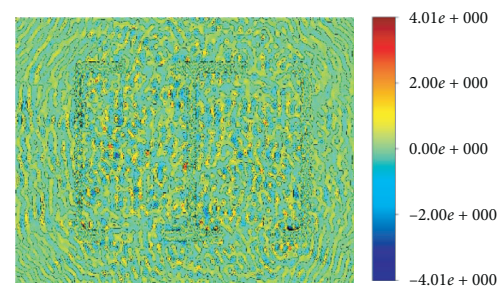

(c)

Figure 7: The isometric (a), $x-y$ plane (b), and $x-z$ plane (c) view of the rooms for $3600 \mathrm{MHz}$.

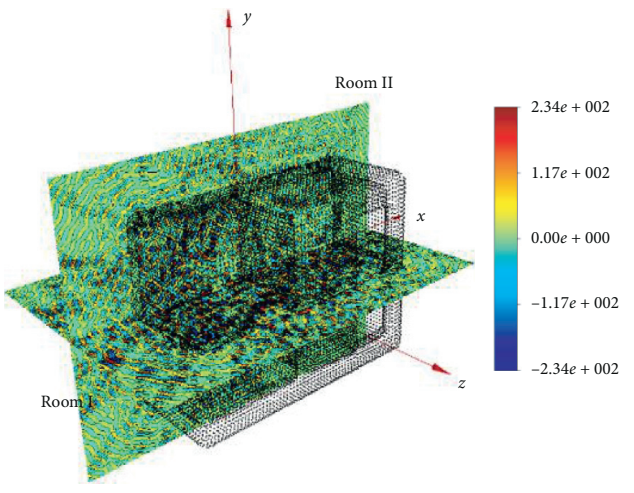

(a)
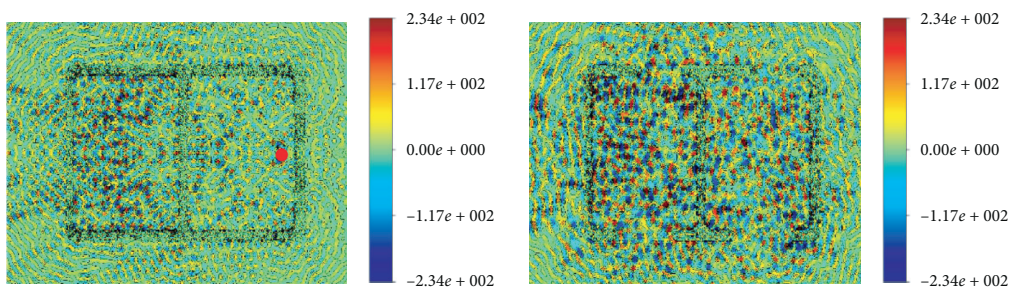

(b) (c)

FIgURe 8: The isometric (a), $x-y$ plane (b), and $x-z$ plane (c) view of the rooms for the source location $(1.2 \mathrm{~m}, 0,0)$.

the first room at which the source is not located has higher field values. The maximum amplitude of the total electric field in the rooms is approximately $2.3 \times 10^{2}[\mathrm{~V} / \mathrm{m}]$.

In Figure 9, the source location is $(1.2 \mathrm{~m}, 0.6 \mathrm{~m}, 0.7 \mathrm{~m})$ which is in the corner of Room II. The electric field distribution is much more uniform for both rooms on the $x-y$ and $x-z$ planes compared to the previous case given in
Figure 8 . The source location is given as a red circle on the figure. The maximum amplitude of the total electric field in the rooms is approximately $1.2 \times 10^{3}[\mathrm{~V} / \mathrm{m}]$.

In Figure 10, the source is located at $(0.3 \mathrm{~m}, 0,0)$, which is in Room II and shown as a red circle. The location is approximately in the middle of the geometry. The result is very common for the coupled-resonator systems. Even 


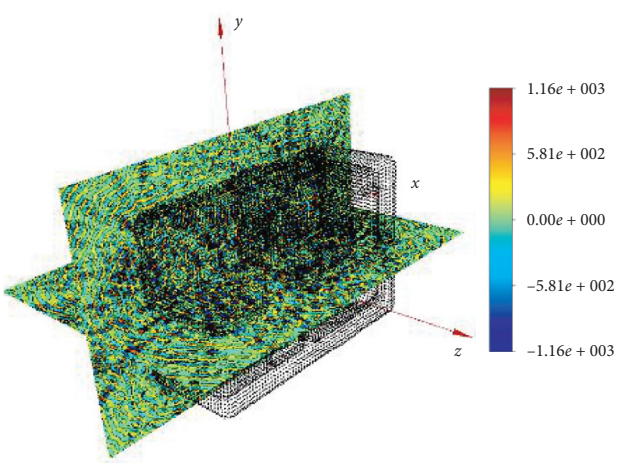

(a)

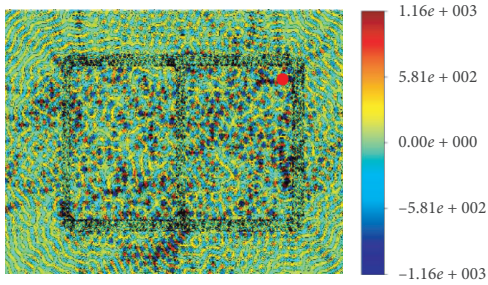

(b)

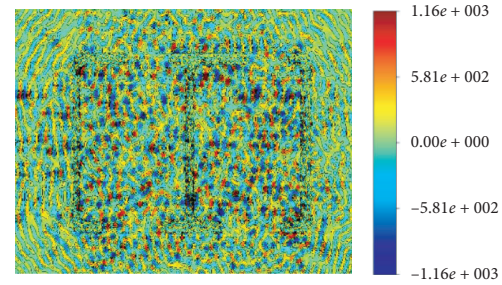

(c)

Figure 9: The isometric (a), $x-y$ plane (b), and $x-z$ plane (c) view of the rooms for the source location $(1.2 \mathrm{~m}, 0.6 \mathrm{~m}, 0.7 \mathrm{~m})$.

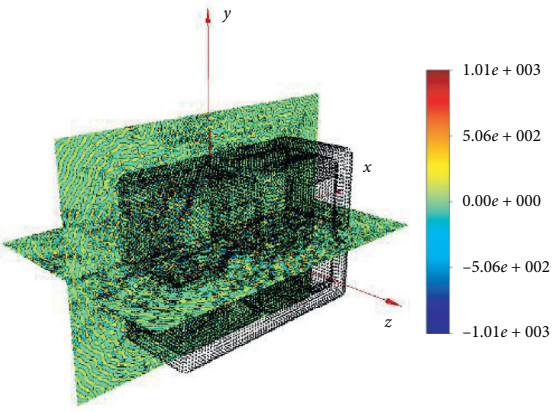

(a)

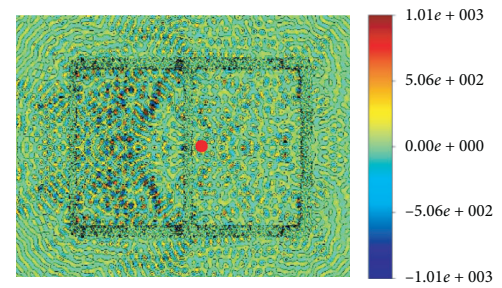

(b)

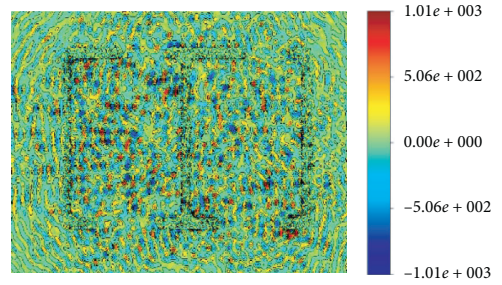

(c)

Figure 10: The isometric (a), $x-y$ plane (b), and $x-z$ plane (c) view of the rooms for the source location $(0.3 \mathrm{~m}, 0,0)$.

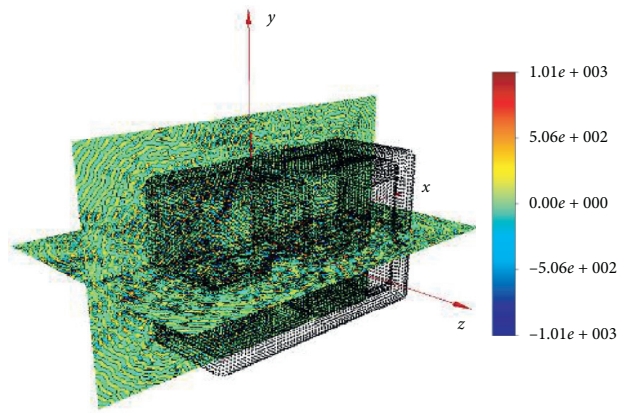

(a)

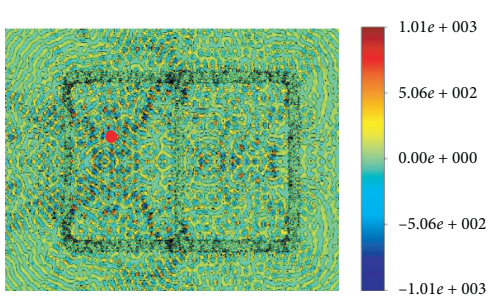

(b)

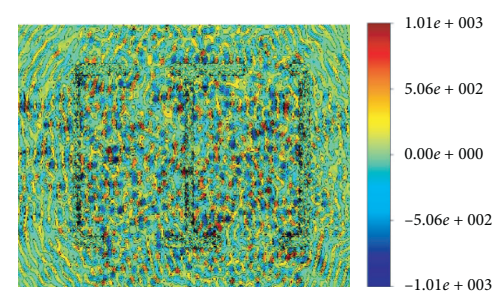

(c)

Figure 11: The isometric (a), $x-y$ plane (b), and $x-z$ plane (c) view of the rooms for the source location $(-0.6 \mathrm{~m}, 0 \mathrm{~m},-0.8 \mathrm{~m})$.

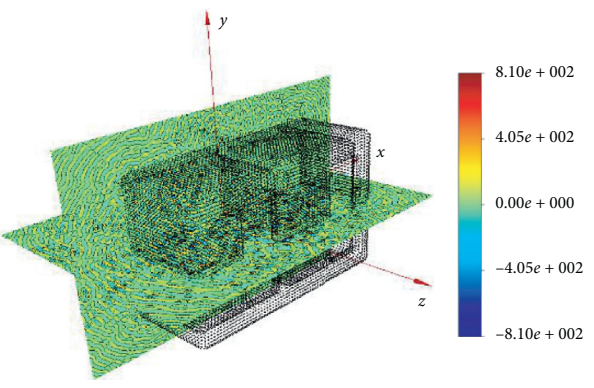

(a)

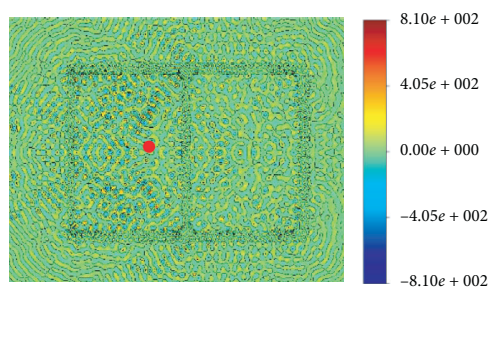

(b)

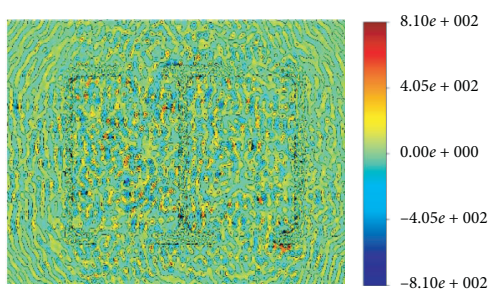

(c)

Figure 12: The isometric (a), $x-y$ plane (b), and $x-z$ plane (c) view of the rooms for the source location $(-0.4 \mathrm{~m}, 0,0.8 \mathrm{~m})$. 
though the field distribution is quite uniform in the $x-z$ plane, the distribution on the other plane is not uniform and has higher values in Room I where the source is not located. The maximum amplitude of the total electric field in the rooms is approximately $1 \times 10^{3}[\mathrm{~V} / \mathrm{m}]$.

In Figure 11, the source is located at $(-0.6 \mathrm{~m}, 0$, $-0.8 \mathrm{~m}$ ) which is near the window in Room I and shown as a red circle. In this case, the field has higher values in Room I also and is uniform on the $x-z$ plane. On the contrary, for the $x-y$ plane, the field is not uniform and its distribution is denser in Room I. The maximum amplitude of the total electric field in the rooms is approximately $1 \times 10^{3}[\mathrm{~V} / \mathrm{m}]$.

In Figure 12, the source is located at $(-0.4 \mathrm{~m}, 0,0.8 \mathrm{~m})$ which is near the door in Room I and shown as a red circle. Again, the field has higher values in Room I for the $x-y$ plane. However mainly, the distribution is nearly uniform for the other plane. The maximum amplitude of the total electric field in the rooms is approximately $8.1 \times 10^{2}[\mathrm{~V} / \mathrm{m}]$.

The Fortran source code to the solution of this problem is available at the link [14].

\section{Conclusion}

In this study, the diffraction of the linearly polarized electromagnetic wave by the building with two rooms is investigated. The problem is solved by using the Method of Auxiliary Sources. The program package is created based on the method. Numerical calculations are carried out for different scenarios. For $5 \mathrm{G}$ frequency, the supercomputer is used. The near electric field distributions are calculated and analyzed based on the representation of the building with two rooms as an opened coupled resonator. The result showed that, for different frequencies or the source locations, the field distribution changed dramatically because of the electromagnetic coupling of the rooms. By changing the location of the source, the coverage differs. Therefore, it is possible to have better coverage for indoor applications by taking into account the source location. For some cases mentioned in the paper, the field distribution of the room which is further away from the source location with respect to the other room has higher field values. This is against the intuition; therefore, it emphasizes the necessity and importance of the studies conducted in the paper. The other method such as ray tracing cannot predict such phenomena. In order to experimentally verify our results, the geometry of the building can be scaled taking into account the electrical length and put into an anechoic chamber for the measurement in order to avoid noise.

\section{Data Availability}

No data were used to support this study.

\section{Conflicts of Interest}

The authors declare that they have no conflicts of interest.

\section{Acknowledgments}

The authors would like to thank the National Center for High-Performance Computing (UHEM) located at Istanbul Technical University for access. This work is partly supported by the Istanbul Technical University (ITU) Vodafone Future Lab under Project ITUVF20180901P10.

\section{References}

[1] T. Jones, Future Agenda: The World in 2020, Infinite Ideas, Oxford, UK, 2010.

[2] Qualcomm, Spectrum for 4G and 5G Licensed Spectrum Spectrum Spectrum, Qualcomm Technologies Inc., San Diego, CA, USA, 2017.

[3] C.-F. Chang-Fa Yang, B.-C. Boau-Cheng $\mathrm{Wu}$, and C.-J. Chuen-Jyi Ko, "A ray-tracing method for modeling indoor wave propagation and penetration," IEEE Transactions on Antennas and Propagation, vol. 46, no. 6, pp. 907-919, 1998.

[4] T. M. Schafer, J. Maurer, and W. Wiesbeck, "Measurement and simulation of radio wave propagation in hospitals," in Proceedings IEEE 56th Vehicular Technology Conference, pp. 792-796, Vancouver, Canada, September 2003.

[5] F. Fuschini, S. Häfner, M. Zoli et al., "Analysis of in-room mm-wave propagation: directional channel measurements and ray tracing simulations," Journal of Infrared, Millimeter, and Terahertz Waves, vol. 38, no. 6, pp. 727-744, 2017.

[6] E. M. Vitucci, J. Chen, V. Degli-Esposti, J. S. Lu, H. L. Bertoni, and $\mathrm{X}$. Yin, "Analyzing radio scattering caused by various building elements using millimeter-wave scale model measurements and ray tracing," IEEE Transactions on Antennas and Propagation, vol. 67, no. 1, pp. 665-669, 2019.

[7] M. B. Tabakcioglu, "Coverage prediction for triple diffraction scenarios," Applied Computational Electromagnetics Society Journal, vol. 33, pp. 1217-1222, 2018.

[8] I. M. Petoev, V. A. Tabatadze, and R. S. Zaridze, "The method of auxiliary sources applied to problems of electromagnetic wave diffraction by certain metal-dielectric structures," Journal of Communications Technology and Electronics, vol. 58, no. 5, pp. 404-416, 2013.

[9] V. Jeladze, V. Tabatadze, M. Prishvin et al., "Human EM exposure study for some big scenarios," in Proceedings of the 2014 IEEE 34th International Scientific Conference on Electronics and Nanotechnology (ELNANO), pp. 342-345, Kyiv, Ukraine, April 2014.

[10] R. Zaridze, G. Bit-Babik, K. Tavzarashvili, D. P. Economou, and N. K. Uzunoglu, "Wave field singularity aspects in largesize scatterers and inverse problems," IEEE Transactions on Antennas and Propagation, vol. 50, no. 1, pp. 50-58, 2002.

[11] V. B. Jeladze, M. M. Prishvin, V. A. Tabatadze, I. M. Petoev, and R. S. Zaridze, "Application of the method of auxiliary sources to study the influence of resonance electromagnetic fields on a man in large spatial domains," Journal of Communications Technology and Electronics, vol. 62, no. 3, pp. 195-204, 2017.

[12] S. Pisa, E. Pittella, E. Piuzzi, P. D’Atanasio, and A. Zambotti, "Permittivity measurement on construction materials through free space method," in Proceedings of the 2017 IEEE International Instrumentation and Measurement Technology Conference (I2MTC), pp. 1-4, Turin, Italy, May 2017.

[13] J. A. Stratton, Electromagnetic Theory, John Wiley \& Sons, Hoboken, NJ, USA, 2007.

[14] GitHub, https://github.com/vasilitabatadze/vasilitabatadze. 